\title{
INFRA-ICE: An ultra-high vacuum experimental station for laboratory astrochemistry
}

Cite as: Rev. Sci. Instrum. 91, 124101 (2020); https://doi.org/10.1063/5.0027920

Submitted: 01 September 2020 . Accepted: 16 November 2020 . Published Online: 02 December 2020

(iD Gonzalo Santoro, (D) Jesús. M. Sobrado, (D) Guillermo Tajuelo-Castilla, Mario Accolla, Lidia Martínez, Jon Azpeitia, (i) Koen Lauwaet, (D) José Cernicharo, (D) Gary J. Ellis, and José Ángel Martín-Gago

\section{ARTICLES YOU MAY BE INTERESTED IN}

A new multi-beam apparatus for the study of surface chemistry routes to formation of complex organic molecules in space

Review of Scientific Instruments 91, 124504 (2020); https://doi.org/10.1063/5.0018926

Determining the deformation and resulting coupling efficiency degradation of ultrastable fiber-coupled optical benches under load

Review of Scientific Instruments 91, 123001 (2020); https://doi.org/10.1063/5.0020776

Bench tests of a helium ion source for the neutral particle diagnostic system of the ITER tokamak

Review of Scientific Instruments 91, 123301 (2020); https://doi.org/10.1063/5.0022149

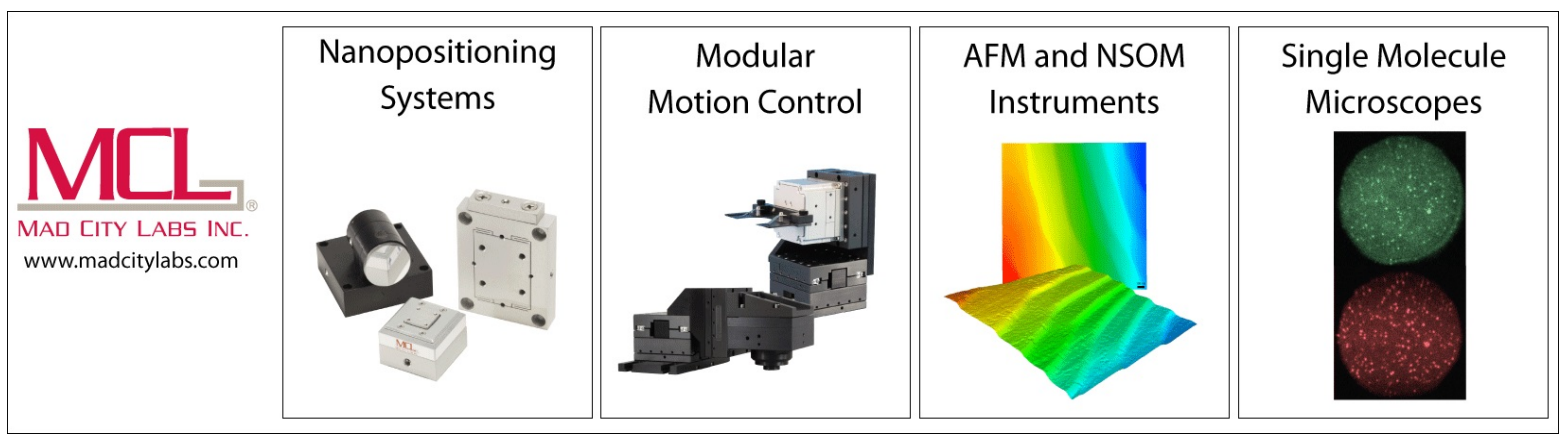




\title{
INFRA-ICE: An ultra-high vacuum experimental station for laboratory astrochemistry
}

\author{
Cite as: Rev. Sci. Instrum. 91, 124101 (2020); doi: 10.1063/5.0027920 \\ Submitted: 1 September 2020 - Accepted: 16 November 2020 • \\ Published Online: 2 December 2020
}

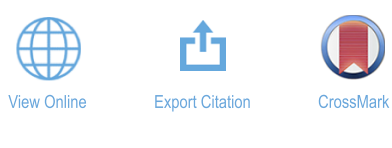

\begin{abstract}
Gonzalo Santoro, ${ }^{1, a)}$ (D) Jesús. M. Sobrado, ${ }^{2}$ (D) Guillermo Tajuelo-Castilla,' (D) Mario Accolla,' Lidia Martínez, Jon Azpeitia, ${ }^{7}$ Koen Lauwaet, ${ }^{3}$ (D) José Cernicharo, ${ }^{4}$ (D) Gary J. Ellis, ${ }^{5}$ (D) and José Ángel Martín-Gago ${ }^{1, a)}$
\end{abstract}

\author{
AFFILIATIONS \\ ${ }^{1}$ Instituto de Ciencia de Materiales de Madrid (ICMM, CSIC), Materials Science Factory, Structure of Nanoscopic Systems \\ Group, c/Sor Juana Inés de la Cruz 3, E-28049 Cantoblanco, Madrid, Spain \\ ${ }^{2}$ Centro de Astrobiología (CAB, INTA-CSIC), Crta. de Torrejón a Ajalvir km 4, E-28850 Torrejón de Ardoz, Madrid, Spain \\ ${ }^{3}$ IMDEA Nanociencia, Ciudad Universitaria de Cantoblanco, E-28049 Cantoblanco, Madrid, Spain \\ ${ }^{4}$ Instituto de Física Fundamental (IFF, CSIC), Group of Molecular Astrophysics, c/Serrano 123, 28006 Madrid, Spain \\ ${ }^{5}$ Instituto de Ciencia y Tecnología de Polímeros (ICTP, CSIC), c/Juan de la Cierva 3, E-28006 Madrid, Spain
}

a) Authors to whom correspondence should be addressed: gonzalo.santoro@icmm.csic.es and gago@icmm.csic.es

\begin{abstract}
Laboratory astrochemistry aims at simulating, in the laboratory, some of the chemical and physical processes that operate in different regions of the universe. Amongst the diverse astrochemical problems that can be addressed in the laboratory, the evolution of cosmic dust grains in different regions of the interstellar medium (ISM) and its role in the formation of new chemical species through catalytic processes present significant interest. In particular, the dark clouds of the ISM dust grains are coated by icy mantles and it is thought that the ice-dust interaction plays a crucial role in the development of the chemical complexity observed in space. Here, we present a new ultra-high vacuum experimental station devoted to simulating the complex conditions of the coldest regions of the ISM. The INFRA-ICE machine can be operated as a standing alone setup or incorporated in a larger experimental station called Stardust, which is dedicated to simulate the formation of cosmic dust in evolved stars. As such, INFRA-ICE expands the capabilities of Stardust allowing the simulation of the complete journey of cosmic dust in space, from its formation in asymptotic giant branch stars to its processing and interaction with icy mantles in molecular clouds. To demonstrate some of the capabilities of INFRA-ICE, we present selected results on the ultraviolet photochemistry of undecane $\left(\mathrm{C}_{11} \mathrm{H}_{24}\right)$ at $14 \mathrm{~K}$. Aliphatics are part of the carbonaceous cosmic dust, and recently, aliphatics and short $n$-alkanes have been detected in situ in the comet 67P/Churyumov-Gerasimenko.
\end{abstract}

Published under license by AIP Publishing. https://doi.org/10.1063/5.0027920

\section{INTRODUCTION}

Laboratory astrophysics and astrochemistry constitute a very powerful tool for investigating the fundamental physical and chemical processes governing the evolution of matter in space. By simulating, in the laboratory, the conditions of different regions of the universe, it is possible not only to test hypotheses derived from astronomical observations and models but also to provide the astronomers with plausible chemical and physical mechanisms that may operate in space, which would help in the correct interpretation of the results derived from observations and physico-chemical modeling. Thus, laboratory astrophysics arises from the interplay between astronomers, physicists, and chemists to synergistically address the chemical evolution of matter in the universe.

Laboratory astrochemistry encompasses diverse topics related to the chemistry of different regions of the universe comprising the gas-phase chemistry of species relevant to the chemical evolution in space, ${ }^{1-5}$ the simulation of planetary atmospheres, ${ }^{6,7}$ the spectroscopic characterization of radicals and ions, ${ }^{8-17}$ and the simulation of the circumstellar envelopes (CSEs) of asymptotic giant branch stars (AGBs) ${ }^{18-21}$ as well as of different interstellar environments, among others.

Among the diverse open questions concerning the development of molecular complexity in space, the role of cosmic dust 
grains deserves particular attention. Cosmic dust is mainly formed in the CSEs of AGBs and is subsequently ejected into the interstellar medium (ISM) and coated by icy mantles in the molecular clouds by the condensation of gas-phase molecules. ${ }^{32}$ During this long journey from the parent star to the ISM, cosmic dust cools down from 1000 to $1500 \mathrm{~K}$ in the dust formation region of the AGBs to around $10 \mathrm{~K}$ in the coldest regions of the ISM. ${ }^{19,33}$ In addition, dust grains are subjected to energetic processing, starting in the outer layers of the star where galactic ultraviolet (UV) photons penetrate the CSE initiating a very rich photochemistry and continuing in the molecular clouds, where the volume density of molecules is $10^{2} \mathrm{~mol} \mathrm{~cm}^{-3}-10^{6} \mathrm{~mol}$ $\mathrm{cm}^{-3}$. From an astrochemical point of view, cosmic dust is believed to actively participate in the synthesis of molecules in space by catalyzing chemical reactions on its surface. ${ }^{34-36}$ However, much is still lacking in the understanding of the exact nature of the catalytic role of dust grains as well as their interactions with icy mantles in dense molecular clouds.

Chemical reactions in the ISM are induced by the processing of matter by cosmic rays and UV photons both in the gas and in the solid phase. ${ }^{37}$ Cosmic rays (protons, nuclei of heavy atoms, alpha particles, and electrons) are ubiquitous throughout the ISM, and in the obscured regions of interstellar clouds where UV photons cannot penetrate, cosmic rays ionize the gaseous species being the gas-phase chemistry dominated by ion-neutral reactions. On the other hand, UV photons reach the edge of interstellar clouds and control the chemistry by destroying most chemical species in these regions and producing new molecules.

In the obscure, cold and dense regions of the ISM most molecules are condensed into the surfaces of dust grains in the form of molecular ices and a very rich chemistry is initiated by the UV field leading to the formation of complex organic molecules. Moreover, the impact of ions on the icy mantles leads to the physico-chemical modification of the ices, which can also promote the formation of new species. ${ }^{38}$ The new molecules synthesized as a consequence of the UV and/or ion processing can be incorporated into to the gas-phase by ion sputtering of the solid material ${ }^{39}$ as well as by the increase in the temperature of the dust/ice system as a result of, e.g., the explosion of a nearby supernova or the increase in temperature in a protoplanetary disk.

The UV photochemistry of molecular ices has been extensively studied in the laboratory. ${ }^{40}$ For instance, the formation of aminoacids has been observed from the simplest, such as glycine, to more complex aminoacids, such as serine and aspartic acid, through the UV irradiation of molecular ice mixtures resembling the composition of interstellar ices. ${ }^{41}$ More recently, the central molecular subunit of RNA, ribose, has been synthesized in the laboratory by UV irradiation of precometary ice analogs. ${ }^{42}$ On the other hand, cosmic rays are highly ionizing radiation and the ions produced by the impact of cosmic rays can, as above-mentioned, interact with dust grains and icy mantles producing sputtering of the material and promoting chemical reactions. ${ }^{38}$ In addition, the interaction of cosmic rays and solid matter induces a cascade of secondary electrons that can participate in ice-grain chemistry (e.g., high energy electron irradiation of silane at low temperature has been shown to promote the formation of $\mathrm{Si}_{n} \mathrm{H}_{2 n+2}$ molecules, therefore inducing the growth of polysilanes, ${ }^{43}$ whereas the exposure of a $\mathrm{CO}_{2}: \mathrm{CH}_{4}: \mathrm{NH}_{3}$ ice mixture to low energy electrons has proved the synthesis of glycine $\left.{ }^{44}\right)$. Finally, surface etching of SiC grains by exposure to atomic hydrogen has been shown to generate large polycyclic aromatic hydrocarbons (PAHs). ${ }^{4}$

To address the catalytic activity of cosmic dust, laboratory studies are mandatory. In this sense, experimental setups specially devoted to investigating the chemical reactions at low temperature on the surface of cosmic dust analogs have been developed. ${ }^{30,46,47}$ Recently, the catalytic effect of carbonaceous cosmic dust analogs in the reaction of ammonia and carbon dioxide has been demonstrated $^{48}$ and the utmost importance of the surface of dust grains on the chemistry when the grains are coated by few monolayers of ice has been emphasized. ${ }^{49}$ This reflects the need for precise and controlled laboratory simulations of the chemistry taking place in the coldest regions of the ISM by the interaction of dust and molecular ices when subjected to processing by UV radiation, ions, and/or electrons. Realistic cosmic dust analogs, i.e., synthesized at conditions resembling those of the dust formation regions of AGBs and processing of their covering ices, are crucial in mimicking the chemistry of the ISM.

Here, we present the INFRA-ICE experimental station that is devoted to investigating the interaction of cosmic dust and molecular ices under conditions resembling those of the coldest regions of ISM, where dust grains are covered with icy mantles. This experimental station can be incorporated as a new module of the Star$d u s t$ machine, ${ }^{20,21,50}$ which is dedicated to simulating, in the laboratory, the complex conditions that lead to the formation of cosmic dust in the CSEs of AGBs, or it can be operated independently as an autonomous experimental station. When incorporated into Stardust, the INFRA-ICE module expands the capabilities of Stardust allowing the study of dust-ice interactions (using dust analogs produced in situ) and the subsequent chemistry that takes place during the processing of these materials in the dark clouds of the ISM. In order to demonstrate some of the capabilities of INFRA-ICE, we present exemplary results on the photoprocessing of undecane $\left(\mathrm{C}_{11} \mathrm{H}_{24}\right)$ at $14 \mathrm{~K}$ as a feasibility study of the UV irradiation of aliphatic hydrocarbons. Aliphatics as part of the carbonaceous cosmic dust have been identified to be widespread in space by its well-known IR absorption bands at wavelengths of $3.4 \mu \mathrm{m}$, $6.8 \mu \mathrm{m}$, and $7.3 \mu \mathrm{m} .{ }^{51-53}$ In addition, aliphatics are known to be present in cometary dust particles, ${ }^{54}$ and, recently, aliphatic hydrocarbons have been detected in situ by the Rosetta mission in the comet $67 \mathrm{P} /$ Churyumov-Gerasimenko, ${ }^{55}$ including short $n$-alkanes (4-5 carbon atoms). ${ }^{56}$

\section{THE INFRA-ICE EXPERIMENTAL STATION}

The INFRA-ICE experimental station is a new ultra-high vacuum (UHV) module that has been incorporated into the Stardust machine. A detailed description of the other Stardust modules can be found elsewhere. ${ }^{50}$ Briefly, the Stardust machine (Fig. 1) is devoted to simulating, in the laboratory, the formation of cosmic dust in the atmosphere of AGB stars and comprises a set of different UHV modules that can be arranged in the most favorable configuration depending on the particular experimental requirements. The first module (MICS) consists of a multiple ion cluster source ${ }^{57,58}$ that allocates three independent magnetron sputter sources. The cosmic dust analogs are synthesized in this module, and a beam 


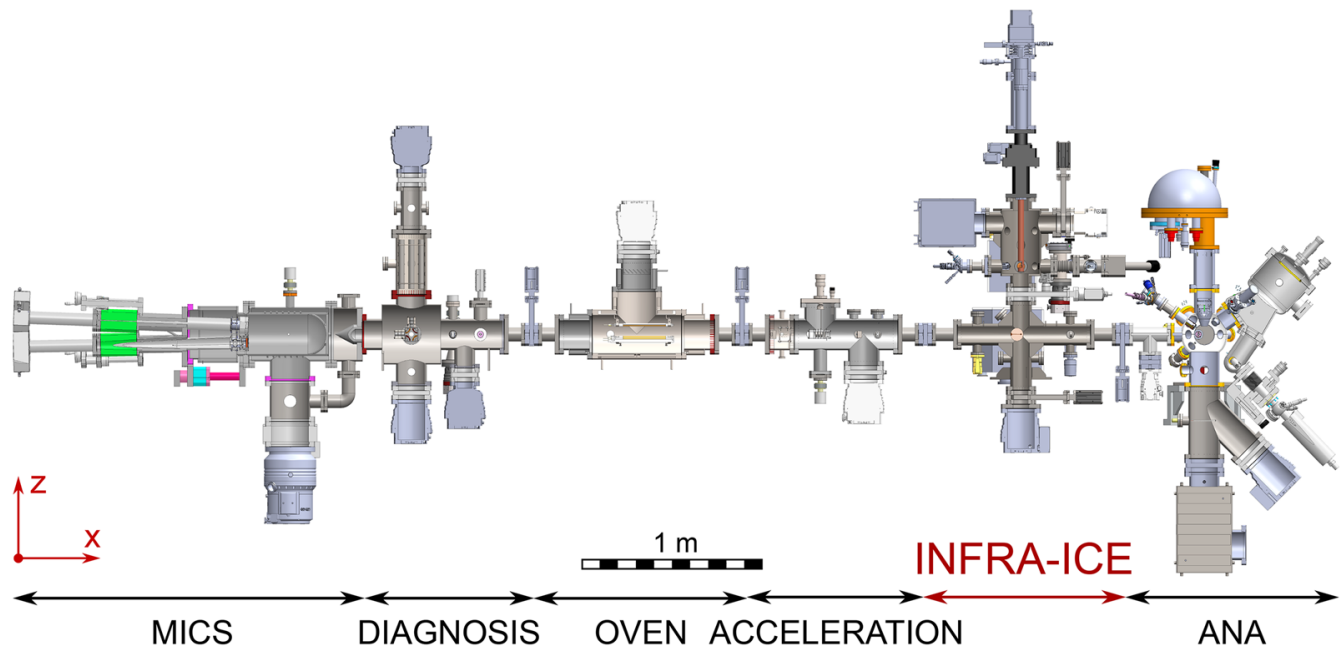

FIG. 1. Side sectional view of the Stardust machine in its standard configuration. The beam of cosmic dust analogs travels along the positive $\mathrm{x}$ direction.

of nanometer-size particles is produced, which travels along the machine. In the standard configuration of Stardust, a module for beam diagnosis is located at the MICS exit. This module (DIAGNOSIS) is dedicated to the characterization of the particle beam properties and accommodates a Faraday cup, a quartz crystal microbalance, and a quadrupole mass spectrometer with a mass range from $0 \mathrm{amu}$ to $10^{6} \mathrm{amu}$, which measure the charge, the production rate, and the mass of the analogs, respectively. The next module (OVEN) is devoted to in-flight heating of the particle beam to temperatures of up to $1400 \mathrm{~K}$ via three $2 \mathrm{~kW}$ infrared lamps. Subsequently, Stardust includes a module (ACCELERATION) to simulate the radiation pressure to which the dust analogs are subjected in the atmosphere of AGBs. This is achieved by ion optics whereby the nanometric dust analogs are ionized, accelerated, and focused by an electron impact ionizer and a set of einzel lenses. The INFRA-ICE module in the standard configuration of Stardust is located between this module and an analysis module (ANA) in which the dust analogs are collected and can be analyzed by electron spectroscopies (x-ray photoelectron spectroscopy, Auger spectroscopy, and ultraviolet photoelectron spectroscopy) and Temperature Programmed Desorption (TPD).

The INFRA-ICE module (base pressure: $1 \times 10^{-10} \mathrm{mbar}$; at low temperature: $5 \times 10^{-11} \mathrm{mbar}$ ) is depicted in Figs. 2(a) $-2(\mathrm{c})$, and a photograph of the setup is shown in Fig. 3. It is a versatile setup that comprises two vertically connected UHV chambers. The lower one is on-axis with the beam of dust analogs produced in Stardust,
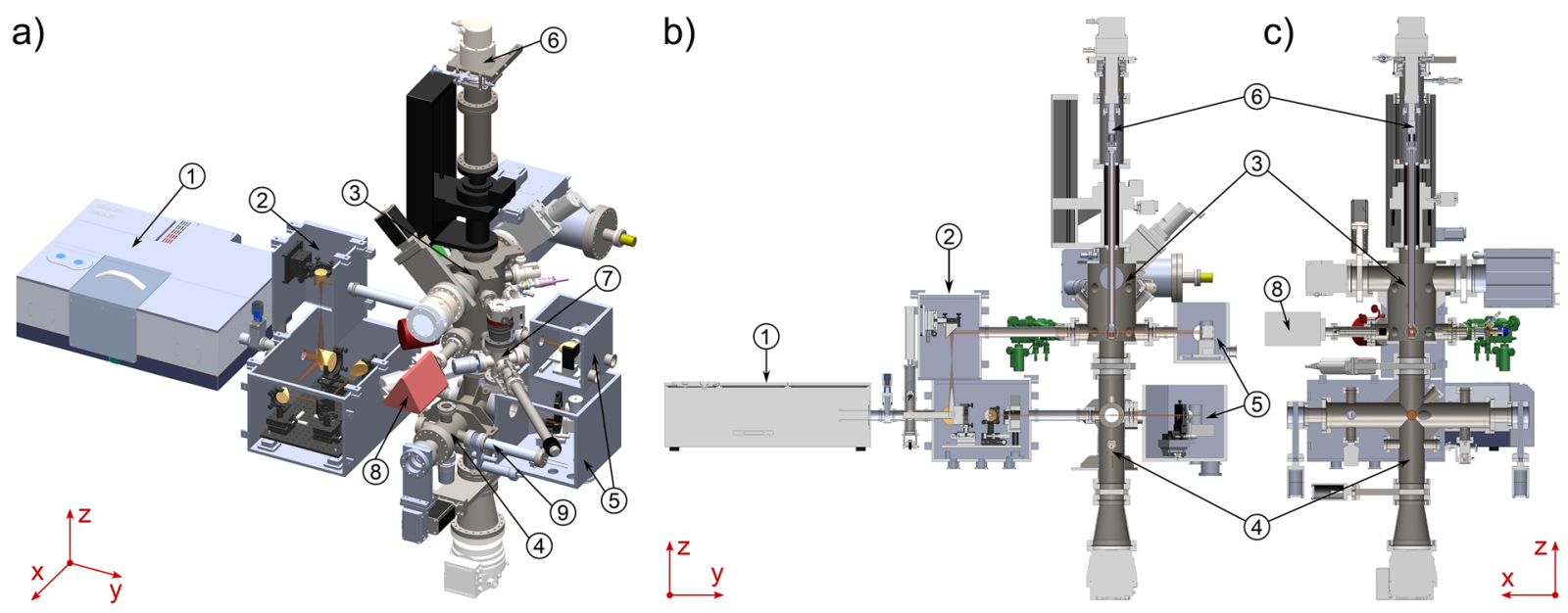

FIG. 2. (a) Isometric view of the INFRA-ICE module. (b) Front and (c) side sectional views. The beam of cosmic dust analogs travels along the positive $x$ direction. (1) IR spectrometer, (2) IR coupling optics, (3) upper UHV chamber, (4) lower UHV chamber, (5) coupling optics for IR detectors, (6) UHV close-cycle helium cryostat, (7) load-lock sample transfer chamber, (8) quadrupole mass spectrometer, and (9) quartz crystal microbalance. 


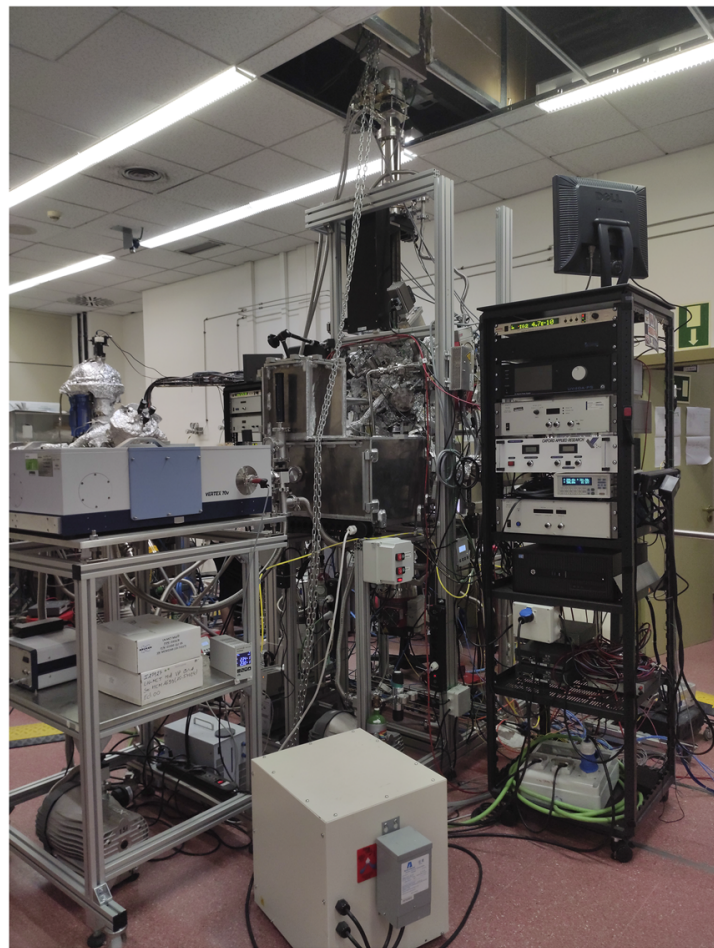

FIG. 3. Photograph of the INFRA-ICE module.

whereas the upper one lies immediately above. Both chambers can be isolated by a UHV gate-valve and have independent vacuum equipment [lower chamber: Bayard-Alpert gauge with tungsten filaments (Lewvac, UK) and turbomolecular pump HiPace 800 (Pfeiffer Vacuum $\mathrm{GmbH}$, Germany); upper chamber: Bayard-Alpert gauge with tungsten filaments (Lewvac, UK), turbomolecular pump HiPace 300 (Pfeiffer Vacuum GmbH, Germany), and ion pump VacIon Plus 150 (Agilent, USA) equipped with a titanium sublimation pump and a cryopanel]. Thus, independent experiments can be carried out in the upper UHV chamber without interrupting the operation of the rest of the Stardust machine. In addition, the lower chamber has UHV gate-valves at both the beam entrance and exit, allowing it to be isolated from the rest of the Stardust machine without interrupting the UHV conditions and operated independently. In this way, the INFRA-ICE module constitutes an autonomous experimental station.

On top of the upper chamber, a UHV close-cycle helium cryostat CCS-UHV/204 (Janis Research, USA) is mounted on a motorized four-axis UHV manipulator (Huntington Mechanical Labs, Inc., USA) comprising three linear (xyz) and one rotation (r) stages with resolutions of $10 \mu \mathrm{m}$ and $0.1^{\circ}$, respectively. The r-axis has a traveling range of $\pm 180^{\circ}$, whereas the $\mathrm{x}$-axis and $\mathrm{y}$-axis have a traveling range of $\pm 9 \mathrm{~mm}$. The traveling range of the $\mathrm{z}$-axis is $400 \mathrm{~mm}$ allowing for transferring the sample from the upper to the lower UHV chambers and vice versa. This enables us to collect the cosmic dust analogs in the lower chamber (which is on-axis with the dust analog beam) and either analyze them by IR spectroscopy, which is the main characterization technique of the INFRA-ICE module, directly at this position, or transfer them to the upper chamber where energetic processing can be performed (see below).

The cryostat operates in the temperature range $13 \mathrm{~K}-300 \mathrm{~K}$ at the sample position with a temperature stability of $0.1 \mathrm{~K}$. Two Si-diode temperature sensors at different positions (at the sample position and $30 \mathrm{~mm}$ above) are used for temperature monitoring, and the temperature is controlled by a LakeShore 335 (Lake Shore Cryotronics, Inc., USA) cryogenic temperature controller. In order to perform transmission and reflectance IR spectroscopy, a modified radiation shield and a sample holder have been fabricated [Fig. 4(a)]. In particular, a sample holder with a $9 \mathrm{~mm}$ diameter hole is used and a cylindrical section has been drilled in the radiation shield, permitting the IR illumination of the sample at the shallow angles needed for performing Infrared Reflection-Absorption Spectroscopy (IRRAS). In addition, a $9 \mathrm{~mm}$ diameter hole has been drilled on the backside of the radiation shield for IR spectroscopy in transmission. The maximum total power irradiated on the sample by the IR sources of the spectrometer has been estimated as $15 \mathrm{~mW}$, which represents a very small heat load and does not affect the temperature stability. These modifications on the radiation shield and sample receiver increase the minimum temperature $(\mathrm{T}=12.6 \mathrm{~K})$ that can be achieved with respect to the cryostat specifications (temperature range according to specifications: $10 \mathrm{~K}-300 \mathrm{~K}$ ). In addition, the long cold finger (length: $780 \mathrm{~mm}$ ) needed to have enough traveling range from the upper to the lower chamber notably increases the cooling time at the sample position [Fig. 4(b)].

IR spectroscopy can be performed in the lower chamber in transmission and reflectance modes (IRRAS) or in the upper chamber in transmission. This is achieved by a properly designed optical path to guide the IR beam from one of the exit ports of the spectrometer to the sample positions inside the chambers [Fig. 5(a)]. All the coupling optical elements are held under vacuum $\left(10^{-1} \mathrm{mbar}\right)$ to a)

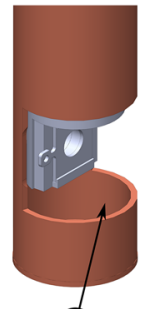

(1)

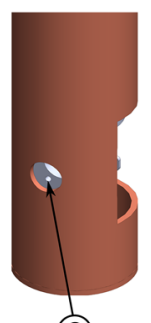

(2)

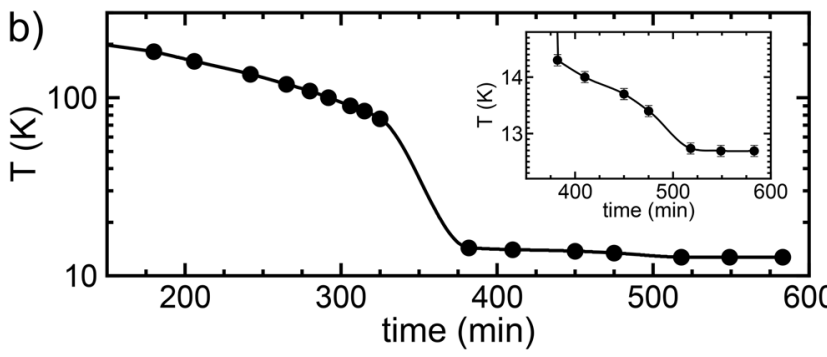

FIG. 4. (a) Radiation shield for the cryostat cold finger at the sample position (1) Front radiation shield optical access and (2) back radiation shield optical access. (b) Temperature evolution at the sample position during cooling down at the maximum cooling power of the cryostat. The inset shows a zoom of the curve. 


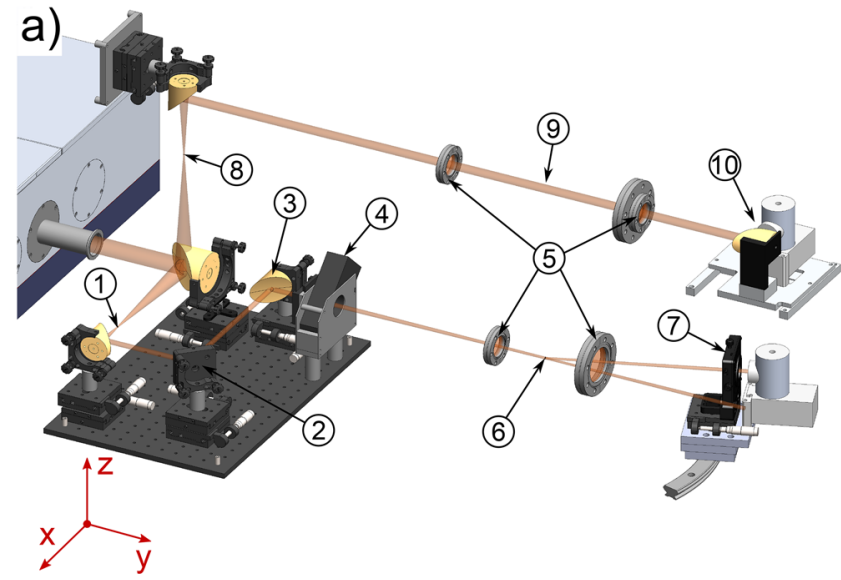

b)

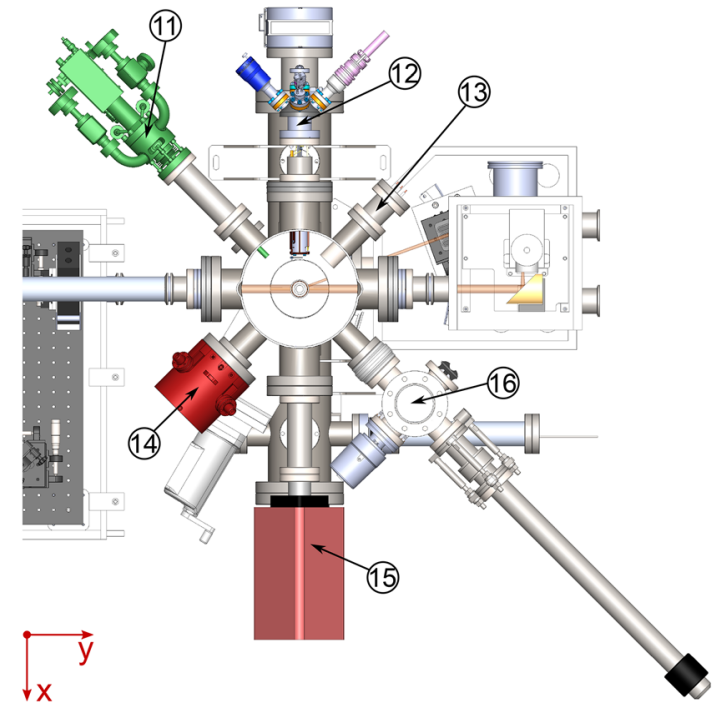

FIG. 5. (a) Radiation shield for the cryostat cold finger at the sample position. (b) Top sectional view of the upper chamber. (1) $4.5 \times$ beam compressor for the lower UHV chamber, (2) flat mirror, (3) focusing mirror for the lower UHV chamber, (4) polarizer and photoelastic modulator, (5) wedged ZnSe windows, (6) sample position in the lower UHV chamber (to represent the optical paths for transmission and reflectance spectroscopy, the IR beam has been split at this position), (7) focusing $\mathrm{ZnSe}$ lens and IR detector, (8) 2.25x beam compressor for the upper UHV chamber, (9) sample position in the upper UHV chamber, (10) focusing mirror and IR detector, (11) UV source, (12) hydrogen cracker, (13) electron gun, (14) ion source, (15) quadrupole mass spectrometer, and (16) load-lock sample transfer chamber.

suppress contributions from atmospheric water and carbon dioxide. To isolate the spectrometer and coupling optics from the UHV of the chambers, we used $\mathrm{ZnSe}$ windows (wedged: $5.8 \mathrm{mrad}$ ) bonded to double-sided CF flanges.

The first element in the optical setup comprises a Vertex $70 \mathrm{~V}$ Fourier Transform IR (FT-IR) spectrometer (Bruker Optik GmbH, Germany) with both the mid-infrared (MIR) and near infrared (NIR) capability, covering a spectral range from $12800 \mathrm{~cm}^{-1}$ $400 \mathrm{~cm}^{-1}(0.78 \mu \mathrm{m}-25 \mu \mathrm{m})$ with a maximum spectral resolution of
$0.16 \mathrm{~cm}^{-1}$. However, the $\mathrm{ZnSe}$ windows present a transmission cutoff starting at $650 \mathrm{~cm}^{-1}$ with no transmission below $500 \mathrm{~cm}^{-1}$, which sets the upper wavelength limit of the spectral range. The MidInfrared (MIR) configuration of the spectrometer comprises two sources (a standard air-cooled globar MIR source and a water-cooled high-power MIR source), a KBr beamsplitter, and two detectors [a DLaTGS detector and a liquid nitrogen cooled Mercury-CadmiumTelluride (MCT) detector]. On the other hand, the NIR configuration consists of a tungsten halogen lamp, $\mathrm{a} \mathrm{CaF}_{2}$ beamsplitter, and an InGaAs-diode detector. A rapid-scan option for performing timeresolved spectroscopy is available at a maximum scanner velocity of $160 \mathrm{kHz}$.

The optical path [Fig. 5(a)] toward the lower UHV chamber consists in, first, a $4.5 \times$ beam compressor that comprises two $90^{\circ}$ off-axis parabolic gold mirrors with focal lengths of $9 \mathrm{in.}$ and $2 \mathrm{in}$., respectively. Due to the shallow angles used for illumination in IRRAS, overillumination (i.e., beam size at a sample position larger than the sample size) is typically found. Thus, this beam compressor provides a better matching between the IR spot size at the sample position and the sample size (standard sample size $10 \times 10 \mathrm{~mm}^{2}$ ). The compressed beam is then reflected by a flat gold mirror that directs the IR beam to a $90^{\circ}$ off-axis gold parabolic mirror with a focal length of $500 \mathrm{~mm}$, which focuses the IR beam at the sample position in the center of the defined dust particle beam axis in the lower UHV chamber. In between this mirror and the ZnSe UHV entrance window, an IR polarizer and a ZnSe photoelastic modulator (PEM) (Hinds Instruments, OR, USA) operating at $50 \mathrm{kHz}$ can be installed enabling Polarization-Modulated IRRAS (PM-IRRAS) measurements to be performed. A detailed description of the PMIRRAS technique can be found elsewhere. ${ }^{59}$ Once the sample is illuminated, the IR beam, either transmitted or reflected, goes through a ZnSe UHV exit window that limits the minimum incident angle for IRRAS to $76^{\circ}$ with respect to the sample surface normal (note that the incident beam is fixed, whereas the incident angle is selected by rotating the sample). Then, a $40 \mathrm{~mm}$ diameter $\mathrm{ZnSe}$ lens is used to focus the IR beam onto the detector. A lens is used instead of a parabolic mirror to maintain the polarization properties of the IR beam required for performing PM-IRRAS. The IR detector and the lens are mounted on a curved guideway to accurately position the detector with respect to the reflected IR beam while maintaining the same sample-lens distance.

To access the upper UHV chamber, the first off-axis parabolic mirror after the spectrometer is rotated by $90^{\circ}$ to guide the IR beam upwards and a $2.25 \times$ beam compressor is found employing a second $90^{\circ}$ off-axis parabolic gold mirror of $4 \mathrm{in}$. focal length. This beam compressor preserves the beam collimation at the exit port of the spectrometer while reducing the spot size to better adjust to the sample size and to the modifications performed on the cryostat sample receiver and radiation shield (see below). The compressed IR beam crosses the $\mathrm{ZnSe}$ entrance window is transmitted through the sample and exits the upper UHV chamber through another ZnSe window. Since, in this chamber, only transmission spectroscopy is performed, a $90^{\circ}$ off-axis parabolic gold mirror is used to focus the beam onto the detector.

To monitor the deposition rate of the dust analogs produced with Stardust, the lower chamber is equipped with a quartz crystal microbalance (QCM). It is mounted on a UHV linear translator with the linear motion in the direction perpendicular to the NP beam. 
In this way, it can be placed in the NP beam axis to monitor the deposition rate and retracted to allow the NP beam to travel toward the next module of Stardust.

The upper chamber allocates a quadrupole mass spectrometer (QMS) PrismaPlus QMG 220 M2 (Pfeiffer Vacuum GmbH, Germany) with a mass range of $1 \mathrm{amu}-200 \mathrm{amu}$. The QMS serves to check the residual gas in the UHV chamber prior to the deposition of molecular ices as well as to monitor the ice deposition process. It also allows temperature programmed desorption measurements as it has the direct view of the sample surface.

In addition, the upper chamber also allocates several processing equipment [Fig. 5(f)], permitting the exposure of the samples to similar processing as those occurring in the interstellar clouds of the ISM. As mentioned in Sec. I, the main energetic processes in molecular clouds are UV radiation and cosmic rays, the latter producing both ions, and a cascade of secondary electrons. The UV field in these regions is dominated by the Lyman- $\alpha$ emission of atomic hydrogen, whereas the ions have energies in the $\mathrm{keV}-$ $\mathrm{MeV}$ range. While high energy ions mainly promote the sputtering of the material, ${ }^{39}$ ions in the $\mathrm{keV}$ range more efficiently lead to physico-chemical changes in the icy mantles that can result in the formation of new chemical species. ${ }^{38}$ On the other hand, most of the large number of secondary electrons produced by cosmic ray impact on icy mantles and dust grains are low energy secondary electrons $(<100 \mathrm{eV})$, which can induce a wide variety of radiation-driven chemical reactions. ${ }^{44,60,61}$ The processing equipment incorporated in INFRA-ICE is mainly dedicated to simulating the main processes inducing chemical changes in the ice-dust system in dense molecular clouds and therefore include the following: (i) a UV source $40 A 2$ (Prevac, Poland) that can be operated with different discharge gases (such as $\mathrm{H}_{2}, \mathrm{He}$, or Ar) so that the UV source can provide different spectral emission profiles and photon energy depending on the discharge gas employed, (ii) an ion source IQP 10/63 (Specs GmbH, Germany) providing ion energies from $0.2 \mathrm{keV}$ to $6 \mathrm{keV}$, and (iii) an electron flood gun $F G$ 15/40 (Specs GmbH, Germany) delivering electrons of energies between $1 \mathrm{eV}$ and $500 \mathrm{eV}$. Moreover, in the diffuse clouds of the ISM, atomic hydrogen is particularly abundant, and to simulate the exposure of cosmic dust to atomic hydrogen in these interstellar regions, the upper chamber also allocates a hydrogen thermal gas cracker TGC-H (Specs GmbH, Germany) for the exposure of the analogs to atomic hydrogen.

Finally, for ice growth, the gases (either pure or gas mixtures) are supplied from a gas mixing system and injected through leak valves located in both the upper and lower chambers. The gas mixing system is thoroughly described in the supplementary material elsewhere. ${ }^{50}$ Briefly, it can accommodate up to four gas bottles and two liquid reservoirs. Both the gases and liquid vapors are introduced in the mixing chamber (base pressure $<10^{-7} \mathrm{mbar}$ ) through calibrated gas-flow valves, and a homogeneous gas mixture is achieved by in-flow mixing via a rotary pump equipped with a zeolite filter.

\section{SELECTED RESULTS: PHOTOCHEMISTRY OF $\mathrm{C}_{11} \mathrm{H}_{24}$ AT LOW TEMPERATURE}

To illustrate some of the capabilities of the INFRA-ICE experimental station, in this section, we present exemplary results on the photochemistry of undecane $\left(\mathrm{C}_{11} \mathrm{H}_{24}\right)$ at $14 \mathrm{~K}$. First, the calibration of the photon flux of the UV lamp employed is presented, which is needed to derive quantitative results for the photochemical experiments.

\section{A. UV lamp flux calibration}

UV irradiation in dense molecular clouds produces a rich photochemistry of the icy mantles that are accreted on cosmic dust particles. The emission from $\mathrm{H}_{2}$ discharge lamps has been shown to satisfactorily reproduce the radiation field of the diffuse interstellar medium ${ }^{62}$ and are, therefore, commonly employed to simulate the UV photoprocessing of interstellar ice analogs. The vacuum UV emission of these lamps is dominated by the Lyman- $\alpha$ emission from atomic hydrogen $(\lambda=121.6 \mathrm{~nm})$ with contributions from molecular hydrogen emission centered at around $\lambda=160 \mathrm{~nm}^{6}$

A common procedure for deriving the photon flux of hydrogen discharge lamps is $\mathrm{O}_{2}$ actinometry. ${ }^{26,64}$ This measures the photochemical conversion of $\mathrm{O}_{2}$ to $\mathrm{O}_{3}$, and through the quantum yield of the reaction, it is possible to convert the number of $\mathrm{O}_{3}$ molecules produced per time unit into photon flux. Usually, the column density (number of molecules per unit area along the observation direction) of $\mathrm{O}_{3}$ is derived from the $v_{3}$ asymmetric stretch of $\mathrm{O}_{3}$ at $1040 \mathrm{~cm}^{-1}$, whose band strength is known for the gas-phase.

However, this method presents several disadvantages. First, $\mathrm{O}_{2}$ has no IR absorption features, and therefore, only the increase in the $v_{3}$ mode of $\mathrm{O}_{3}$ is observed during actinometric measurements. More importantly, even if the solid-phase quantum yield and solidphase band strength data for the $v_{3}$ mode of $\mathrm{O}_{3}$ are reported in the literature, ${ }^{65,66}$ these depend on the structure of the $\mathrm{O}_{2}$ and $\mathrm{O}_{3}$ ices. Finally, the optimum UV range in which $\mathrm{O}_{2}$ photolysis takes place is from $130 \mathrm{~nm}$ to $190 \mathrm{~nm},{ }^{67}$ which covers most of the UV emission of molecular hydrogen but excludes the Lyman- $\alpha$ line of atomic hydrogen.

For these reasons, other actinometric systems (e.g., the CO photoproduction from $\mathrm{CO}_{2}{ }^{68}$ ) have been employed, although for these systems solid-phase quantum-yield data are usually not available. The use of silicate photodiodes ${ }^{69}$ and metallic meshes ${ }^{63}$ avoids these problems, but an accurate calibration of the devices in the vacuum UV region is needed, which requires an already calibrated lamp, not always available. A straightforward method for flux calibration was developed by Fulvio et al. using the photocurrent generated in a gold photodetector.

To calibrate our UV lamp, we have used the photolysis of methane, which has been thoroughly investigated in ices, and accurate photodestruction cross section data in the far UV spectral range are available in the literature. ${ }^{64,69,70}$ This method relies on measuring the photolysis rate and is, therefore, independent of the band strength of the IR bands used; thus, it can even be applied to IR bands with unknown band strength.

The photolysis of an optically thin molecular ice can be described by the first-order reaction kinetics, ${ }^{69}$

$$
\frac{d n}{d t}=-k n,
$$

where $n$ is the number density of molecules and $k$ is the photolysis rate, which is dependent on both the photodestruction cross section $\sigma(\lambda)$ and the photon flux $I(\lambda)$. Assuming these two values to 
be constant over the wavelength range of interest, which is a reasonable approximation for narrow spectral ranges, ${ }^{69} k$ can be simply expressed as the product of $\sigma$ and $I$. Thus, by measuring the temporal evolution of the IR bands corresponding to the molecule being photolyzed, the incident photon flux is easily derived.

Figure 6(a) shows the IR spectra of $\mathrm{CH}_{4}$ ice at different UV exposure times. The experiments were performed in the upper chamber of the INFRA-ICE experimental station. The $\mathrm{CH}_{4}$ ice was deposited at $14 \mathrm{~K}$ on IR transparent $\mathrm{KBr}$ substrates through the so-called background deposition method. ${ }^{71}$ In our discharge lamp, the UV emission is guided through a windowless quartz capillary. Windowless discharge lamps have the advantage of avoiding photon flux losses due to the deposition on the windows of the material generated in the discharge, a phenomenon that is commonly observed when working with windowed discharge lamps. ${ }^{62}$ These deposits produce an increase in the absorption of the window over time and, therefore, a decrease of the photon flux on the sample during irradiation. ${ }^{69}$ However, in the case of windowless lamps, a flow of the discharge gas is introduced into the chamber, which might interact with the sample. For the photolysis experiments, our UV lamp was operated with a flow of $\mathrm{H}_{2}$ (purity 99.99\%) that resulted in a pressure in the upper chamber of INFRA-ICE of $2 \times 10^{-8}$ mbar. Therefore, the experiments were performed in an enriched $\mathrm{H}_{2}$
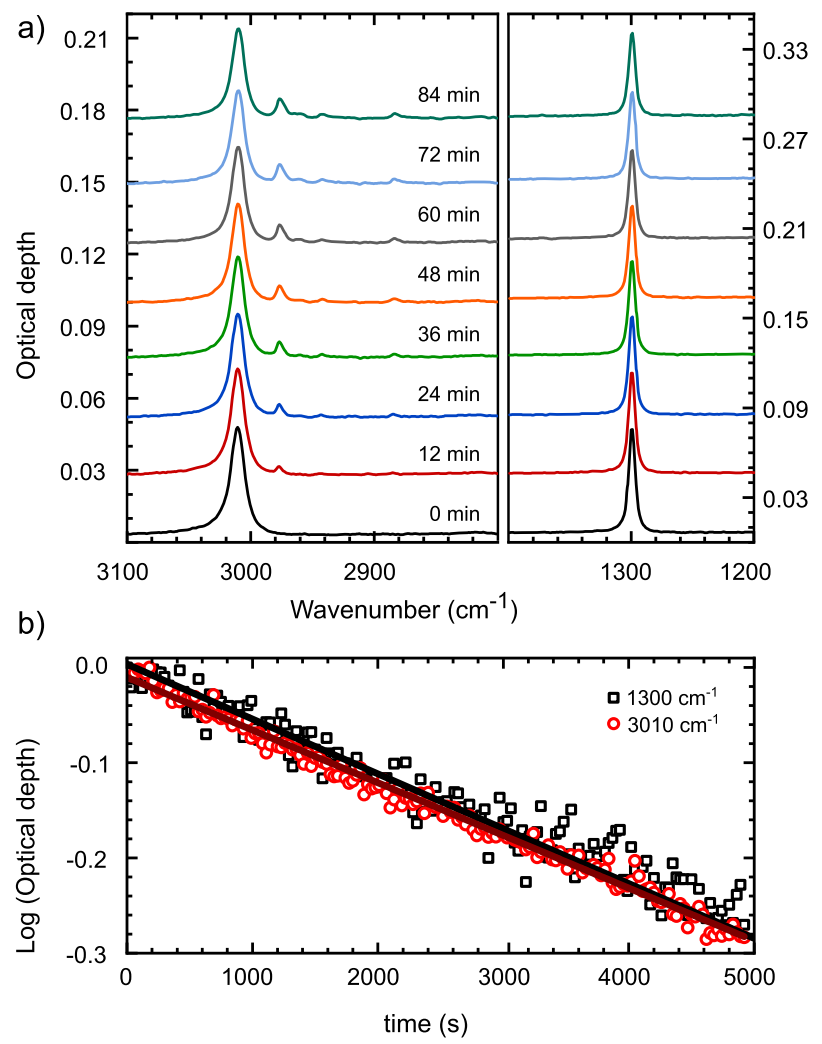

FIG. 6. (a) IR absorption spectra during the photolysis of $\mathrm{CH}_{4}$ ice. The irradiation time of each spectrum is indicated, and the curves have been shifted for clarity. (b) Temporal evolution of the optical depth for the $1300 \mathrm{~cm}^{-1}$ and $3010 \mathrm{~cm}^{-1}$ bands. The solid lines correspond to the linear fit of the data. atmosphere. However, at $14 \mathrm{~K} \mathrm{H}_{2}$ does not condense and, thus, $\mathrm{H}_{2}$ was not deposited on the sample. According to the manufacturer, these $\mathrm{H}_{2}$ flow conditions maximize the emission of the Lyman- $\alpha$ line over the emission of molecular hydrogen. A power of $60 \mathrm{~W}$ was applied to the discharge. During the UV exposure, IR spectra were concurrently recorded every $30 \mathrm{~s}$ with a spectral resolution of $2 \mathrm{~cm}^{-1}$ and 64 scans were coadded for each spectrum.

The spectra of pure $\mathrm{CH}_{4}$ ice present two distinct absorption bands at $1300 \mathrm{~cm}^{-1}$ and $3010 \mathrm{~cm}^{-1}$, which correspond to the $\mathrm{C}-\mathrm{H}$ bending and $\mathrm{C}-\mathrm{H}$ stretching vibrational modes of $\mathrm{CH}_{4}$, respectively. As the ice is irradiated, both bands decrease in intensity and new bands appear in the range $3000 \mathrm{~cm}^{-1}-2800 \mathrm{~cm}^{-1}$, which are due to the $\mathrm{CH}_{2}$ and $\mathrm{CH}_{3}$ stretching modes of $\mathrm{C}_{2} \mathrm{H}_{6}$ and $\mathrm{C}_{3} \mathrm{H}_{8}$. The production of larger alkanes from the vacuum UV exposure of $\mathrm{CH}_{4}$ is a well-known process involving the photolysis of $\mathrm{CH}_{4}$ to $\mathrm{CH}_{2}$ $+\mathrm{H}_{2}$ and to $\mathrm{CH}_{3}+\mathrm{H}^{7}$

Figure 6(b) shows the temporal evolution of the natural logarithm of the integrated optical depth (normalized to the maximum value) of the $1300 \mathrm{~cm}^{-1}$ and $3010 \mathrm{~cm}^{-1}$ bands of $\mathrm{CH}_{4}$ during UV exposure. Linear fittings to the data yield photolysis rates of $(5.76$ $\pm 0.07) \times 10^{-5} \mathrm{~s}^{-1}$ and $(5.53 \pm 0.04) \times 10^{-5} \mathrm{~s}^{-1}$ for the $1300 \mathrm{~cm}^{-1}$ and $3010 \mathrm{~cm}^{-1}$ bands, respectively. These correspond to a photon flux (integrated over the complete UV spectral range) of $(6.2 \pm 0.6)$ $\times 10^{14} \mathrm{ph} \mathrm{s}^{-1} \mathrm{~cm}^{-2}$, using a photodestruction cross section of 9.1 $\times 10^{-20} \mathrm{~cm}^{2}$ for $\mathrm{CH}_{4}{ }^{69}$ and considering the mean value of both derived photolysis rates.

\section{B. UV photochemistry of $\mathrm{C}_{11} \mathrm{H}_{24}$}

Recently, by simulating the circumstellar envelope of carbonrich AGBs in the laboratory with the Stardust machine, we have shown that the interaction of atomic carbon with hydrogen, the latter being the most abundant gaseous species in AGBs, leads predominantly to aliphatics, including alkanes. ${ }^{20}$ Moreover, as mentioned in the Introduction, aliphatics are widespread in space as the component of the carbonaceous cosmic dust ${ }^{52,53}$ and are also present in cometary dust particles. ${ }^{54}$ Also recently, aliphatic hydrocarbons have been identified in situ by the Rosetta mission in the comet 67P/Churyumov-Gerasimenko, ${ }^{55}$ including short $n$-alkanes of 4-5 carbon atoms in the gas-phase. ${ }^{56}$ In this section, to show some of the capabilities of INFRA-ICE, we present the results obtained during the UV exposure of undecane $\left(\mathrm{C}_{11} \mathrm{H}_{24}\right)$ as a feasibility study of the UV photochemistry of aliphatic hydrocarbons at low temperatures.

The experiments were performed in the upper chamber of INFRA-ICE, where the $\mathrm{C}_{11} \mathrm{H}_{24}$ vapor was deposited at $14 \mathrm{~K}$ on $\mathrm{KBr}$ substrates and exposed to UV radiation for $240 \mathrm{~min}$. For the deposition, $\mathrm{C}_{11} \mathrm{H}_{24}$ (purity: $\geqslant 99 \%$ ) was loaded into a Pyrex ampoule with conflat fittings and warmed to $345 \mathrm{~K}$. The UV lamp was loaded with $\mathrm{H}_{2}$ (purity: 99.99\%), and the hydrogen discharge was carried out under the same conditions as those described in Sec. III A. Thus, the total UV exposure corresponded to a photon fluence of $\sim 9 \times 10^{18}$ photons $\mathrm{cm}^{-2}$. Throughout the complete UV treatment, IR spectra were simultaneously acquired every $190 \mathrm{~s}$ in the transmission mode with a spectral resolution of $2 \mathrm{~cm}^{-1}$, coadding 128 scans for each spectrum.

The IR spectra of $\mathrm{C}_{11} \mathrm{H}_{24}$, and of $n$-alkanes and aliphatics in general, are dominated by the absorptions in the spectral region $3000 \mathrm{~cm}^{-1}-2800 \mathrm{~cm}^{-1}$, which correspond to the $\mathrm{CH}_{2}$ and $\mathrm{CH}_{3}$ 
stretching modes [see Fig. 7(a)]. Additionally, the bands at around $1380 \mathrm{~cm}^{-1}$ along with those at around $1460 \mathrm{~cm}^{-1}$ are ascribed to the highly distinctive $\mathrm{CH}_{3}$ symmetric bending ("umbrella" deformation) mode and to the $\mathrm{CH}_{3}$ asymmetric bending/ $\mathrm{CH}_{2}$ scissoring modes, respectively, and are very characteristic of aliphatics. In the case of $n$-alkanes, the position of the $\mathrm{CH}_{3}$ symmetric bending mode and the band structure of the absorptions at around $1460 \mathrm{~cm}^{-1}$ are highly sensitive to the conformational structure of the molecules ${ }^{72}$ and are, therefore, very sensitive to crystallinity. For $n$ alkanes, the crystalline phase is comprised exclusively of $\mathrm{CH}_{2}-\mathrm{CH}_{2}$ trans conformers, whereas gauche conformers are associated with the amorphous material.

In our case, the IR spectrum of as-deposited $\mathrm{C}_{11} \mathrm{H}_{24}$ reflects the amorphous structure of the material as can be derived from the shape, position, and width of the bands at $1378 \mathrm{~cm}^{-1}$ and in the $1420 \mathrm{~cm}^{-1}-1480 \mathrm{~cm}^{-1}$ region. ${ }^{72,73}$ The amorphous structure of $\mathrm{C}_{11} \mathrm{H}_{24}$ is related to the temperature of the $\mathrm{KBr}$ substrate where the ice is grown. Figure 7 (a) shows the IR spectra of non-irradiated $\mathrm{C}_{11} \mathrm{H}_{24}$ and after an UV exposure of $240 \mathrm{~min}$. Clear changes are
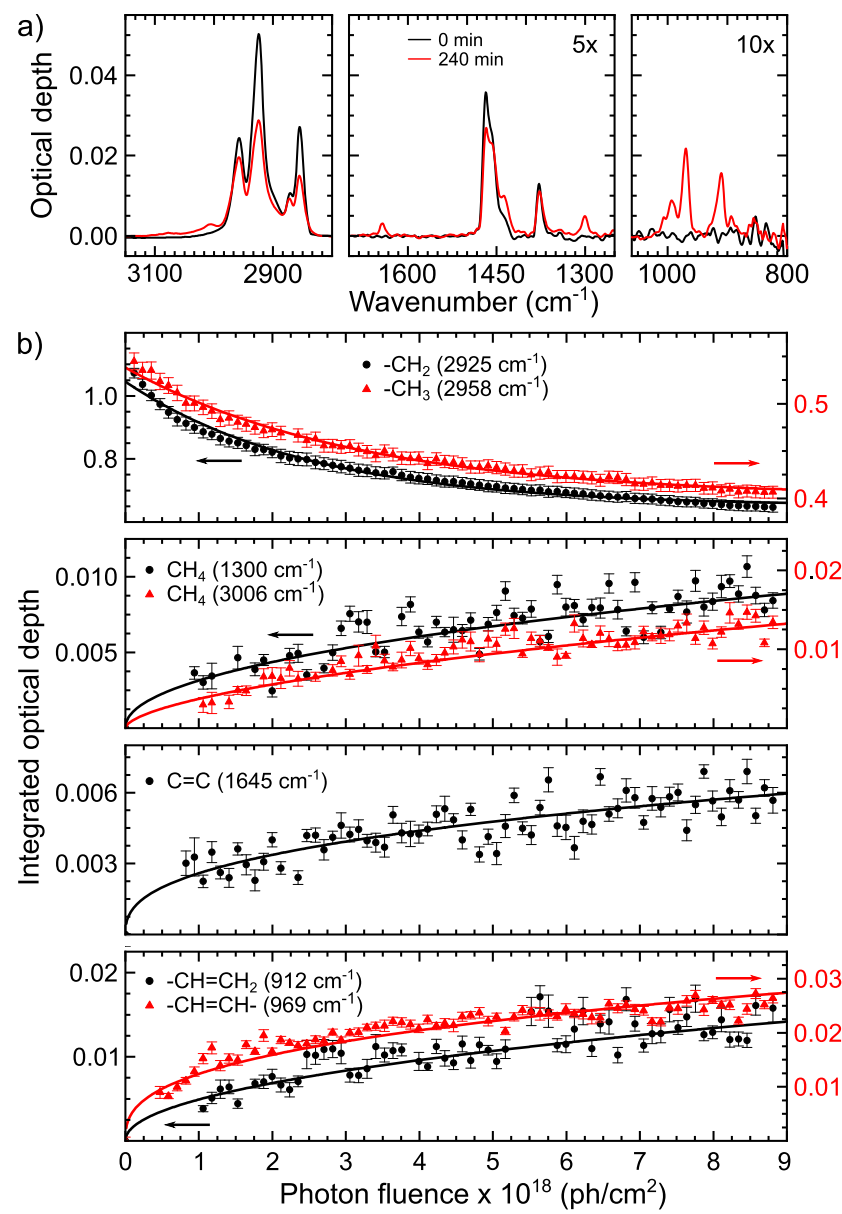

FIG. 7. (a) IR spectra of $\mathrm{C}_{11} \mathrm{H}_{24}$ as deposited and after 240 min of UV exposure. (b) Evolution of the optical depth of selected IR bands during UV exposure. The arrows indicate the $y$-axis for each curve, whereas the solid lines are guides to the eye. observed after the UV irradiation, which consist mainly in a reduction in absorption in the $\mathrm{CH}_{2}$ and $\mathrm{CH}_{3}$ stretching modes and the appearance of new absorption bands. The positions and assignments of the observed IR bands are listed in Table I. The evolution of some selected IR bands during the UV photoprocessing of $\mathrm{C}_{11} \mathrm{H}_{24}$ is shown in Fig. 7(b). The integrated optical depth for each absorption feature has been obtained by band deconvolution using Gaussian curves.

It can be observed that the decrease in the intensity of the $\mathrm{CH}_{2}$ and $\mathrm{CH}_{3}$ stretching modes follows an exponential decay corresponding to the first order reaction kinetics as expected for a photolysis process [the symmetric $\mathrm{CH}_{2}$ and $\mathrm{CH}_{3}$ stretching modes at $2925 \mathrm{~cm}^{-1}$ and $2958 \mathrm{~cm}^{-1}$, respectively, are shown in Fig. 5(b)]. Additionally, the evolution of the bands at around $1460 \mathrm{~cm}^{-1}$ presents a complex behavior. In particular, the band at $1470 \mathrm{~cm}^{-1}$, which can be ascribed to $\mathrm{CH}_{2}$ moieties, decreases in the same way as the $\mathrm{CH}_{3}$ and $\mathrm{CH}_{2}$ stretching modes. On the other hand, the shoulder at $1437 \mathrm{~cm}^{-1}$ is observed to increase. This might be related to the formation of unsaturated hydrocarbon moieties (see below) since a band at around $1440 \mathrm{~cm}^{-1}$ can be assigned to the bending mode of methylene moieties in the presence of adjacent unsaturated groups. ${ }^{74}$ However, a deep interpretation of the spectral changes observed in this spectral region is difficult since, in addition to the formation of new chemical species, the UV exposure of alkanes can induce a conformational rearrangement that produces changes in the band structure of the absorptions at around $1460 \mathrm{~cm}^{-1}$, which, as above-mentioned, is very sensitive to the conformational structure.

On the other hand, the new IR bands at $1300 \mathrm{~cm}^{-1}$ and $3006 \mathrm{~cm}^{-1}$ reveal the production of methane. The release of $\mathrm{CH}_{2}$ and $\mathrm{CH}_{3}$ from the photolysis of $\mathrm{C}_{11} \mathrm{H}_{24}$ promotes the interaction of these with $\mathrm{H}_{2}$ and $\mathrm{H}$ (both released from the photolysis of undecane and from the $\mathrm{H}_{2}$ in the chamber) to form $\mathrm{CH}_{4}$, a process that is

TABLE I. IR band assignment.

\begin{tabular}{|c|c|c|}
\hline Wavenumber $\mathrm{cm}^{-1}$ & Wavelength $\mu \mathrm{m}$ & Assignment $^{\mathrm{a}, \mathrm{b}}$ \\
\hline \multicolumn{3}{|c|}{ As-deposited $\mathrm{C}_{11} \mathrm{H}_{24}$} \\
\hline 2957 & 3.38 & $v_{a s} \mathrm{CH}\left(\mathrm{CH}_{3}\right)$ \\
\hline 2925 & 3.42 & $v_{a s} \mathrm{CH}\left(\mathrm{CH}_{2}\right)$ \\
\hline 2871 & 3.48 & $v_{s} \mathrm{CH}\left(\mathrm{CH}_{3}\right)$ \\
\hline 2854 & 3.50 & $v_{s} \mathrm{CH}\left(\mathrm{CH}_{3}\right)$ \\
\hline 1470 & 6.80 & $\delta_{b} \mathrm{CH}\left(\mathrm{CH}_{2}\right)$ \\
\hline 1458 & 6.86 & $\delta_{s c} \mathrm{CH}\left(\mathrm{CH}_{2}\right), \delta_{a s} \mathrm{CH}\left(\mathrm{CH}_{3}\right)$ \\
\hline 1437 & 6.96 & $\delta_{s c} \mathrm{CH}\left(\mathrm{CH}_{2}\right), \delta_{a s} \mathrm{CH}\left(\mathrm{CH}_{3}\right)$ \\
\hline 1378 & 7.26 & $\delta_{s} \mathrm{CH}\left(\mathrm{CH}_{3}\right)$ \\
\hline \multicolumn{3}{|c|}{ New bands after UV processing of $\mathrm{C}_{11} \mathrm{H}_{24}$} \\
\hline 3076 & 3.25 & $v_{a s} \mathrm{CH}(=\mathrm{C}-\mathrm{H})$ \\
\hline 3006 & 3.33 & $v_{a s} \mathrm{CH}\left(\mathrm{CH}_{4}\right)$ \\
\hline 1645 & 6.08 & $v \mathrm{C}=\mathrm{C}$ \\
\hline 1300 & 7.69 & $\delta \mathrm{CH}\left(\mathrm{CH}_{4}\right)$ \\
\hline 994 & 10.06 & $\gamma_{\text {oop }} \mathrm{CH}\left(-\mathrm{CH}=\mathrm{CH}_{2}\right)_{v i n y l}$ \\
\hline 967 & 10.34 & $\gamma_{\text {oop }} \mathrm{CH}(-\mathrm{HC}=\mathrm{CH}-)_{\text {trans }}$ \\
\hline 912 & 10.96 & $\gamma_{\text {oop }} \mathrm{CH}\left(-\mathrm{CH}=\mathrm{CH}_{2}\right)_{\text {vinyl }}$ \\
\hline
\end{tabular}

${ }^{\mathrm{a}}$ The vibrational modes are abbreviated as follows: $v$ : stretching; $\delta$ : deformation (b: bend; sc: scissor); $\gamma$ : wagging; s: symmetric; as: asymmetric; oop: out-of-plane.

${ }^{\mathrm{b}}$ Assignments from Refs. 70 and $72-74$. 
well-known for the photolysis of alkanes. ${ }^{75}$ More interestingly, as the UV irradiation proceeds, a new infrared band appears at $1645 \mathrm{~cm}^{-1}$, which is assigned to the $\mathrm{C}=\mathrm{C}$ stretching mode of alkenes, ${ }^{74}$ proving the formation of unsaturated hydrocarbons. In addition, a very weak band at $3076 \mathrm{~cm}^{-1}$ can be observed in the spectrum of the UV irradiated material in Fig. 7(a). This band is assigned to the $\mathrm{CH}$ stretching of olefins. $^{74}$

The nature of the alkenes formed by photoprocessing is revealed by the absorption features in the spectral region corresponding to deformation vibrations of $\mathrm{C}-\mathrm{H}\left(1000 \mathrm{~cm}^{-1}\right.$ $800 \mathrm{~cm}^{-1}$ ). In particular, the bands at $994 \mathrm{~cm}^{-1}$ and $912 \mathrm{~cm}^{-1}$ are very characteristic of vinyl moieties, whereas the band at $967 \mathrm{~cm}^{-1}$ is characteristic of trans-vinylene hydrocarbons. ${ }^{74}$ Therefore, the UV photoprocessing of $\mathrm{C}_{11} \mathrm{H}_{24}$ promotes the formation of olefinic moieties both at the end and in the backbone of the chains in the form of vinyl $\left(-\mathrm{CH}=\mathrm{CH}_{2}\right)$ and vinylene chemical groups $(-\mathrm{CH}=\mathrm{CH}-)$, respectively.

The formation of $\mathrm{CH}_{4}$ and unsaturated hydrocarbons is further confirmed by TPD measurements during the sublimation of the UV-exposed $\mathrm{C}_{11} \mathrm{H}_{24}$ ice. To conduct the TPD measurements, two different procedures were employed. An initial TPD measurement was performed using multiple ion detection at selected $\mathrm{m} / \mathrm{z}$ signals, whereas a second TPD measurement was carried out acquiring the complete mass spectra of the desorbed gases in the range $\mathrm{m} / \mathrm{z}$ $=0-200$. The latter allowed for a deeper characterization of the desorbed chemical species. Both procedures were performed on identical UV-exposed $\mathrm{C}_{11} \mathrm{H}_{24}$ samples (UV fluence $\sim 9 \times 10^{18}$ photons $\mathrm{cm}^{-2}$ ) and, for comparison purposes, on identical $\mathrm{C}_{11} \mathrm{H}_{24}$ deposits without UV exposure. A heating rate of $5 \mathrm{~K} \mathrm{~min}^{-1}$ was used in all cases.

Figures 8 (a) and 8 (b) show the evolution of the signal of some selected $\mathrm{m} / \mathrm{z}$ values during the thermal desorption of neat and UVexposed $\mathrm{C}_{11} \mathrm{H}_{24}$ molecular ices, respectively. The $\mathrm{m} / \mathrm{z}$ values were selected according to the electron-impact dissociation patterns of the chemical species that were expected to be formed after the UV processing. In particular, $\mathrm{m} / \mathrm{z}=15$ and 16 are characteristic of $\mathrm{CH}_{4} ; \mathrm{m} / \mathrm{z}$ $=43,57,71$, and 85 correspond to the strongest signals of $\mathrm{C}_{11} \mathrm{H}_{24}$; and $\mathrm{m} / \mathrm{z}=70$ and 83 are characteristic for long chain alkenes, ${ }^{76}$ though they also present contributions from $\mathrm{C}_{11} \mathrm{H}_{24}$. Except the clear desorption of $\mathrm{CH}_{4}$ with a maximum at around $53 \mathrm{~K}$, no evidences of new chemical species were detected by the multiple ion detection procedure, not even by comparison with the non-irradiated $\mathrm{C}_{11} \mathrm{H}_{24}$ ice. In fact, the mass spectra of the unprocessed and UV-exposed samples at $209 \mathrm{~K}$ (which corresponds to the temperature of the maximum desorption of $\mathrm{C}_{11} \mathrm{H}_{24}$ ) are identical and resemble that of $\mathrm{C}_{11} \mathrm{H}_{24}$ in the gas-phase [Fig. 8(c)].

However, important changes can be observed at $170 \mathrm{~K}$ [Fig. 8(d)]. This is the temperature at which the desorption of $\mathrm{C}_{11} \mathrm{H}_{24}$ begins, and therefore, at this temperature, the signal coming from new chemical compounds (which desorb in the same temperature range and with mass peaks overlapping with those of undecane) is not obscured by the much more intense signal from the desorption of $\mathrm{C}_{11} \mathrm{H}_{24}$ at higher temperatures. In comparison to the non-irradiated $\mathrm{C}_{11} \mathrm{H}_{24}$ ice, the mass spectrum of $\mathrm{UV}$-treated $\mathrm{C}_{11} \mathrm{H}_{24}$ exhibits clear differences at $170 \mathrm{~K}$ in the regions at $\mathrm{m} / \mathrm{z}=53-57, \mathrm{~m} / \mathrm{z}$ $=67-71$, and $\mathrm{m} / \mathrm{z}=81-85$, among others. In particular, the peak structure of the mass spectrum of the UV-treated ice is present in these regions fragmentation patterns typical of alkenes ${ }^{76}$ for which
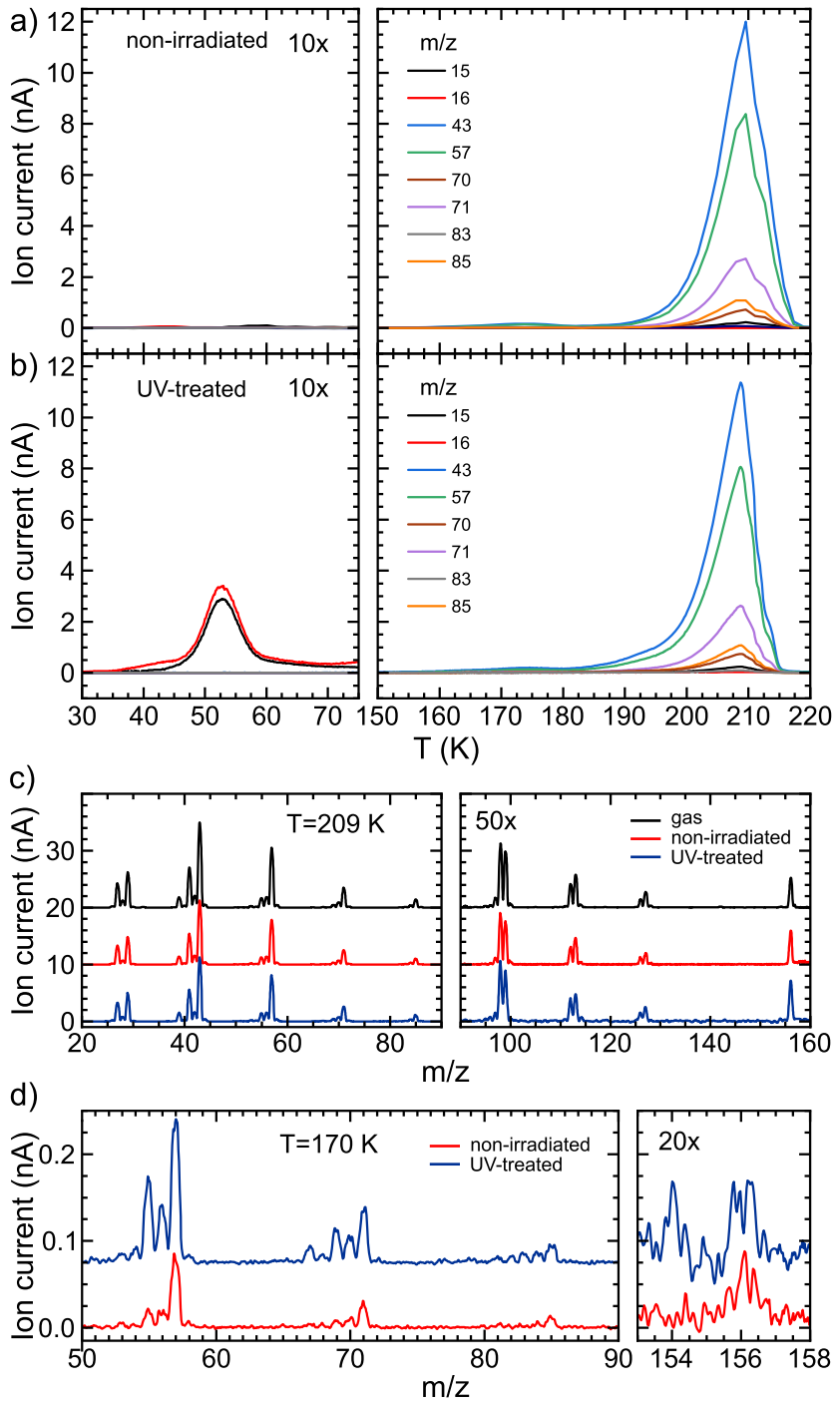

FIG. 8. Evolution of some selected $\mathrm{m} / \mathrm{z}$ peaks (a) during the TPD of non-irradiated $\mathrm{C}_{11} \mathrm{H}_{24}$ and (b) after $240 \mathrm{~min}$ of UV exposure. (c) Mass spectra of the species desorbed at $209 \mathrm{~K}$ for the as-deposited and UV exposed $\mathrm{C}_{11} \mathrm{H}_{24}$. For comparison, the mass spectrum of $\mathrm{C}_{11} \mathrm{H}_{24}$ in the gas-phase is included. The curves have been vertically shifted for clarity. (d) Mass spectra of the species desorbed at $170 \mathrm{~K}$ for the non-irradiated and UV treated $\mathrm{C}_{11} \mathrm{H}_{24}$. The curves have been vertically shifted for clarity.

the maxima in the electron impact fragmentation pattern is lowered in steps by $\mathrm{m} / \mathrm{z}=1$ and 2 regarding the fully saturated counterpart. ${ }^{76}$ Finally, a very faint peak can be appreciated at $\mathrm{m} / \mathrm{z}=154$, which can be ascribed to $\mathrm{C}_{11} \mathrm{H}_{22}$.

\section{CONCLUSIONS}

We have presented a new experimental station devoted to simulating, in the laboratory, the complex conditions of the coldest regions of the ISM and investigating the interaction of cosmic dust with ices of astrophysical interest. The INFRA-ICE experimental 
station is a versatile UHV setup that can be operated independently or integrated as a module of the Stardust machine, which is devoted to simulating, in the laboratory, the formation and evolution of cosmic dust in the circumstellar envelopes of asymptotic giant branch stars. When incorporated into Stardust, INFRA-ICE expands the capabilities of Stardust allowing for the simulation, in the laboratory, of the complete journey of cosmic dust from its formation in evolved stars to its processing in the densest regions of the ISM, where cosmic dust is coated with molecular ices. The main analysis technique of INFRA-ICE is IR spectroscopy (both transmission and reflectance), and a quadrupole mass spectrometer with the direct view of the sample can be employed to perform Temperature Programmed Desorption (TPD) measurements. In addition, a set of processing equipment (UV source, ion gun, electron gun, and hydrogen gas cracker) is available for exposing the samples to similar processes as those encountered in dense molecular clouds.

As an example of the capabilities of the INFRA-ICE experimental station, we have presented the UV photochemistry of $\mathrm{C}_{11} \mathrm{H}_{24}$ at low temperature. We have shown that the UV processing of $\mathrm{C}_{11} \mathrm{H}_{24}$ at $14 \mathrm{~K}$ promotes the formation of unsaturated hydrocarbon species along with the production of methane. In the near future, realistic cosmic dust analogs and their interaction with ices of astrophysical interest will be investigated with the INFRA-ICE experimental station.

\section{ACKNOWLEDGMENTS}

We thank the European Research Council for funding support under Synergy Grant No. ERC-2013-SyG, G.A. 610256 (NANOCOSMOS). In addition, partial support from the Spanish Research Agency (AEI) through Grant Nos. MAT2017-85089c2-1R and FIS2016-77578-R is acknowledged. Support from the FotoArt-CM Project (No. P2018/NMT 4367) through the Program of R\&D activities between research groups in Technologies 2013, co-financed by European Structural Funds, is also acknowledged. G.T.-C. acknowledges funding from the Comunidad Autónoma de Madrid (Grant No. PEJD-2018-PRE/IND-9029). G.S. and G.J.E. would like to thank Stephane Lefrançois for valuable discussions on the mechanical details of the optical coupling.

\section{DATA AVAILABILITY}

The data that support the findings of this study are available from the corresponding author upon reasonable request.

\section{REFERENCES}

${ }^{1}$ A. Canosa, I. R. Sims, D. Travers, I. W. M. Smith, and B. R. Rowe, "Reactions of the methylidine radical with $\mathrm{CH}_{4}, \mathrm{C}_{2} \mathrm{H}_{2}, \mathrm{C}_{2} \mathrm{H}_{4}, \mathrm{C}_{2} \mathrm{H}_{6}$, and but-1-ene studied between 23 and $295 \mathrm{~K}$ with a CRESU apparatus," Astron. Astrophys. 323, 644-651 (1997).

${ }^{2}$ M. Antiñolo, M. Agúndez, E. Jiménez, B. Ballesteros, A. Canosa, G. E. Dib, J. Albaladejo, and J. Cernicharo, "Reactivity of $\mathrm{OH}$ and $\mathrm{CH}(3) \mathrm{OH}$ between 22 and $64 \mathrm{~K}$ : modelling the gas phase production of $\mathrm{CH}(3) \mathrm{O}$ in Barnard 1B," Astrophys. J. 823, 25 (2016).

${ }^{3}$ A. Potapov, A. Canosa, E. Jiménez, and B. Rowe, "Uniform supersonic chemical reactors: 30 years of astrochemical history and future challenges," Angew. Chem., Int. Ed. 56, 8618-8640 (2017).

${ }^{4}$ I. Tanarro, B. Alemán, P. de Vicente, J. D. Gallego, J. R. Pardo, G. Santoro, K. Lauwaet, F. Tercero, A. Díaz-Pulido, E. Moreno, M. Agúndez, J. R. Goicoechea,
J. M. Sobrado, J. A. López, L. Martínez, J. L. Doménech, V. J. Herrero, J. M. Hernández, R. J. Peláez, J. A. López-Pérez, J. Gómez-González, J. L. Alonso, E. Jiménez, D. Teyssier, K. Makasheva, M. Castellanos, C. Joblin, J. A. MartínGago, and J. Cernicharo, "Using radio astronomical receivers for molecular spectroscopic characterization in astrochemical laboratory simulations: A proof of concept," Astron. Astrophys. 609, A15 (2018).

${ }^{5}$ J. Cernicharo, J. D. Gallego, J. A. López-Pérez, F. Tercero, I. Tanarro, F. Beltrán, P. de Vicente, K. Lauwaet, B. Alemán, E. Moreno, V. J. Herrero, J. L. Doménech,

S. I. Ramírez, C. Bermúdez, R. J. Peláez, M. Patino-Esteban, I. López-Fernández,

S. García-Álvaro, P. García-Carreño, C. Cabezas, I. Malo, R. Amils, J. Sobrado,

C. Diez-González, J. M. Hernandéz, B. Tercero, G. Santoro, L. Martínez,

M. Castellanos, B. Vaquero Jiménez, J. R. Pardo, L. Barbas, J. A. López-Fernández,

B. Aja, A. Leuther, and J. A. Martín-Gago, "Broad-band high-resolution rotational spectroscopy for laboratory astrophysics," Astron. Astrophys. 626, A34 (2019).

${ }^{6}$ J. M. Sobrado, J. Martín-Soler, and J. A. Martín-Gago, "Mimicking mars: A vacuum simulation chamber for testing environmental instrumentation for mars exploration," Rev. Sci. Instrum. 85, 035111 (2014).

${ }^{7}$ J. M. Sobrado, J. Martín-Soler, and J. A. Martín-Gago, “Mimicking martian dust: An in-vacuum dust deposition system for testing the ultraviolet sensors on the curiosity rover,” Rev. Sci. Instrum. 86, 105113 (2015).

${ }^{8}$ D. Romanini, L. Biennier, F. Salama, A. Kachanov, L. J. Allamandola, and F. Stoeckel, "Jet-discharge cavity ring-down spectroscopy of ionized polycyclic aromatic hydrocarbons: Progress in testing the pah hypothesis for the diffuse interstellar band problem," Chem. Phys. Lett. 303, 165-170 (1999).

${ }^{9}$ P. Bréchignac and T. Pino, "Electronic spectra of cold gas phase PAH cations: Towards the identification of the Diffuse Interstellar Bands carriers," Astron. Astrophys. 343, L49-L52 (1999).

${ }^{10}$ C. Joblin, C. Pech, M. Armengaud, P. Frabel, and P. Boissel, “A piece of interstellar medium in the laboratory: The PIRENEA experiment,” EAS Publ. Ser. 4, 73 (2002).

${ }^{11}$ L. Biennier, F. Salama, L. J. Allamandola, and J. J. Scherer, "Pulsed discharge nozzle cavity ringdown spectroscopy of cold polycyclic aromatic hydrocarbon ions," J. Chem. Phys. 118, 7863-7872 (2003).

${ }^{12}$ R. I. Kaiser and Y. Osamura, "Infrared spectroscopic studies of hydrogenated silicon clusters. Guiding the search for $\mathrm{Si}_{2} \mathrm{H}_{x}$ species in the Circumstellar Envelope of IRC+10216," Astron. Astrophys. 432, 559-566 (2005).

${ }^{13}$ F. Useli-Bacchitta, A. Bonnamy, G. Mulas, G. Malloci, D. Toublanc, and C. Joblin, "Visible photodissociation spectroscopy of PAH cations and derivatives in the PIRENEA experiment," Chem. Phys. 371, 16-23 (2010).

${ }^{14}$ O. Asvany, F. Bielau, D. Moratschke, J. Krause, and S. Schlemmer, "Note: New design of a cryogenic linear radio frequency multipole trap," Rev. Sci. Instrum. 81, 076102 (2010).

${ }^{15}$ E. K. Campbell and J. P. Maier, "Perspective: $\mathrm{C}^{+}{ }_{60}$ and laboratory spectroscopy related to diffuse interstellar bands," J. Chem. Phys. 146, 160901 (2017).

${ }^{16}$ J. L. Doménech, S. Schlemmer, and O. Asvany, "Accurate rotational rest frequencies for ammonium ion isotopologues,” Astrophys. J. 866, 158 (2018).

${ }^{17}$ J. M. Fernández, G. Tejeda, M. Carvajal, and M. L. Senent, "New spectral characterization of dimethyl ether isotopologues $\mathrm{CH}_{3} \mathrm{OCH}_{3}$ and ${ }^{13} \mathrm{CH}_{3} \mathrm{OCH}_{3}$ in the THz region," Astrophys. J. Suppl. Ser. 241, 13 (2019).

${ }^{18}$ C. Jäger, F. Huisken, H. Mutschke, I. L. Jansa, and T. Henning, "Formation of polycyclic aromatic hydrocarbons and carbonaceous solids in gas-phase condensation experiments," Astrophys. J. 696, 706-712 (2009).

${ }^{19}$ C. S. Contreras and F. Salama, "Laboratory investigations of polycyclic aromatic hydrocarbon formation and destruction in the circumstellar outflows of carbon stars," Astrophys. J. Suppl. Ser. 208, 6 (2013).

${ }^{20}$ L. Martínez, G. Santoro, P. Merino, M. Accolla, K. Lauwaet, J. Sobrado, H. Sabbah, R. J. Pelaez, V. J. Herrero, I. Tanarro, M. Agúndez, A. Martín-Jimenez, R. Otero, G. J. Ellis, C. Joblin, J. Cernicharo, and J. A. Martín-Gago, "Prevalence of non-aromatic carbonaceous molecules in the inner regions of circumstellar envelopes," Nat. Astron. 4, 97-105 (2020).

${ }^{21}$ G. Santoro, L. Martínez, K. Lauwaet, M. Accolla, G. Tajuelo-Castilla, P. Merino, J. M. Sobrado, R. J. Peláez, V. J. Herrero, I. Tanarro, Á. Mayoral, M. Agúndez, H. Sabbah, C. Joblin, J. Cernicharo, and J. Á. Martín-Gago, "The chemistry of cosmic dust analogs from $\mathrm{C}, \mathrm{C}_{2}$, and $\mathrm{C}_{2} \mathrm{H}_{2}$ in C-rich circumstellar envelopes," Astrophys. J. 895, 97 (2020). 
${ }^{22}$ J. E. Roser, G. Vidali, G. Manicò, and V. Pirronello, "formation of carbon dioxide by surface reactions on ices in the interstellar medium," Astrophys. J. 555, L61-L64 (2001).

${ }^{23}$ V. Mennella, G. A. Baratta, M. E. Palumbo, and E. A. Bergin, "Synthesis of CO and $\mathrm{CO}_{2}$ molecules by UV irradiation of water ice-covered hydrogenated carbon grains," Astrophys. J. 643, 923-931 (2006).

${ }^{24}$ Y. Oba, N. Miyauchi, H. Hidaka, T. Chigai, N. Watanabe, and A. Kouchi, "Formation of compact amorphous $\mathrm{H}_{2} \mathrm{O}$ ice by codeposition of hydrogen atoms with oxygen molecules on grain surfaces," Astrophys. J. 701, 464-470 (2009).

${ }^{25}$ M. E. Palumbo, G. A. Baratta, G. Leto, and G. Strazzulla, "H bonds in astrophysical ices," J. Mol. Struct. 972, 64-67 (2010).

${ }^{26}$ G. M. Muñoz Caro, A. Jiménez-Escobar, J. Á. Martín-Gago, C. Rogero, C. Atienza, S. Puertas, J. M. Sobrado, and J. Torres-Redondo, "New results on thermal and photodesorption of $\mathrm{CO}$ ice using the novel InterStellar Astrochemistry Chamber (ISAC)," Astron. Astrophys. 522, A108 (2010).

${ }^{27} \mathrm{H}$. Linnartz, S. Ioppolo, and G. Fedoseev, "Atom addition reactions in interstellar ice analogues," Int. Rev. Phys. Chem. 34, 205-237 (2015).

${ }^{28}$ D. Fulvio, G. Sandor, C. Jager, K. Akos, and T. Henning, "Laboratory experiments on the low-temperature formation of carbonaceous grains in the ISM," Astrophys. J. Suppl. Ser. 233, 11 (2017).

${ }^{29}$ R. L. Hudson, M. J. Loeffler, and K. M. Yocum, "Laboratory investigations into the spectra and origin of propylene oxide: A chiral interstellar molecule," Astrophys. J. 835, 225 (2017).

${ }^{30}$ A. Potapov, C. Jäger, and T. Henning, "Photodesorption of water ice from dust grains and thermal desorption of cometary ices studied by the INSIDE experiment," Astrophys. J. 880, 12 (2019).

${ }^{31}$ Y. Oba, Y. Takano, H. Naraoka, N. Watanabe, and A. Kouchi, "Nucleobase synthesis in interstellar ices," Nat. Commun. 10, 4413 (2019).

${ }^{32}$ A. C. A. Boogert, P. A. Gerakines, and D. C. B. Whittet, "Observations of the Icy Universe," Annu. Rev. Astron. Astrophys. 53, 541-581 (2015).

${ }^{33}$ G. Pascoli and A. Polleux, "Condensation and growth of hydrogenated carbon clusters in carbon-rich stars," Astron. Astrophys. 359, 799-810 (2000).

${ }^{34}$ G. Vidali, " $\mathrm{H}_{2}$ formation on interstellar grains," Chem. Rev. 113, 8762-8782 (2013).

${ }^{35}$ D. A. Williams and C. Cecchi-Pestellini, "Chapter 8 catalysis on the surfaces of bare dust grains," in The Chemistry of Cosmic Dust (The Royal Society of Chemistry, 2016), pp. 157-196

${ }^{36}$ V. Wakelam, E. Bron, S. Cazaux, F. Dulieu, C. Gry, P. Guillard, E. Habart, L. Hornekær, S. Morisset, G. Nyman, V. Pirronello, S. D. Price, V. Valdivia, G. Vidali, and N. Watanabe, " $\mathrm{H}_{2}$ formation on interstellar dust grains: The viewpoints of theory, experiments, models and observations," Mol. Astrophys. 9, 1-36 (2017).

${ }^{37}$ L. J. Allamandola, M. P. Bernstein, S. A. Sandford, and R. L. Walker, "Evolution of interstellar ices," Space Sci. Rev. 90, 219-232 (1999).

${ }^{38}$ G. Strazzulla, G. A. Baratta, and M. E. Palumbo, "Vibrational spectroscopy of ion-irradiated ices," Spectrochim. Acta, Part A 57, 825-842 (2001).

${ }^{39}$ R. E. Johnson, "Sputtering and desorption from icy surfaces," in Solar System Ices, edited by B. Schmitt, C. De Bergh, and M. Festou (Springer Netherlands, Dordrecht, 1998), pp. 303-334.

${ }^{40} \mathrm{~K}$. I. Öberg, "Photochemistry and astrochemistry: Photochemical pathways to interstellar complex organic molecules," Chem. Rev. 116, 9631-9663 (2016).

${ }^{41}$ G. M. Muñoz Caro, U. J. Meierhenrich, W. A. Schutte, B. Barbier, A. Arcones Segovia, H. Rosenbauer, W. H.-P. Thiemann, A. Brack, and J. M. Greenberg, "Amino acids from ultraviolet irradiation of interstellar ice analogues," Nature 416, 403-406 (2002).

${ }^{42}$ C. Meinert, I. Myrgorodska, P. de Marcellus, T. Buhse, L. Nahon, S. V. Hoffmann, L. L. S. d'Hendecourt, and U. J. Meierhenrich, "Ribose and related sugars from ultraviolet irradiation of interstellar ice analogs," Science 352, 208-212 (2016).

${ }^{43}$ G. Tarczay, M. Förstel, P. Maksyutenko, and R. I. Kaiser, "Formation of higher silanes in low-temperature silane $\left(\mathrm{SiH}_{4}\right)$ ices," Inorg. Chem. 55, 8776-8785 (2016).

${ }^{44}$ S. Esmaili, A. D. Bass, P. Cloutier, L. Sanche, and M. A. Huels, "Glycine formation in $\mathrm{CO}_{2}: \mathrm{CH}_{4}: \mathrm{NH}_{3}$ ices induced by $0-70 \mathrm{eV}$ electrons," J. Chem. Phys. 148, 164702 (2018)
${ }^{45}$ P. Merino, M. Švec, J. I. Martinez, P. Jelinek, P. Lacovig, M. Dalmiglio, S. Lizzit, P. Soukiassian, J. Cernicharo, and J. A. Martin-Gago, "Graphene etching on SiC grains as a path to interstellar polycyclic aromatic hydrocarbons formation," Nat. Commun. 5, 3054 (2014).

${ }^{46}$ H. J. Fraser and E. F. van Dishoeck, "Surfreside: A novel experiment to study surface chemistry under interstellar and protostellar conditions," Adv. Space Res. 33, 14-22 (2004).

${ }^{47}$ S. Ioppolo, G. Fedoseev, T. Lamberts, C. Romanzin, and H. Linnartz, "SURFRESIDE2: An ultrahigh vacuum system for the investigation of surface reaction routes of interstellar interest," Rev. Sci. Instrum. 84, 073112 (2013).

${ }^{48}$ A. Potapov, P. Theulé, C. Jäger, and T. Henning, "Evidence of surface catalytic effect on cosmic dust grain analogs: The ammonia and carbon dioxide surface reaction," Astrophys. J. 878, L20 (2019).

${ }^{49}$ A. Potapov, C. Jäger, and T. Henning, "Ice coverage of dust grains in cold astrophysical environments," Phys. Rev. Lett. 124, 221103 (2020).

${ }^{50}$ L. Martínez, K. Lauwaet, G. Santoro, J. M. Sobrado, R. J. Peláez, V. J. Herrero, I. Tanarro, G. J. Ellis, J. Cernicharo, C. Joblin, Y. Huttel, and J. A. Martín-Gago, "Precisely controlled fabrication, manipulation and in-situ analysis of $\mathrm{Cu}$ based nanoparticles," Sci. Rep. 8, 7250 (2018).

${ }^{51}$ J. E. Chiar, A. G. G. M. Tielens, D. C. B. Whittet, W. A. Schutte, A. C. A. Boogert, D. Lutz, E. F. van Dishoeck, and M. P. Bernstein, "The composition and distribution of dust along the line of sight toward the galactic center," Astrophys. J. 537, 749-762 (2000)

${ }^{52}$ Y. J. Pendleton and L. J. Allamandola, "The organic refractory material in the diffuse interstellar medium: Mid-infrared spectroscopic constraints," Astrophys. J. Suppl. Ser. 138, 75-98 (2002).

${ }^{53}$ B. Günay, M. G. Burton, M. Afşar, and T. W. Schmidt, "A method for mapping the aliphatic hydrocarbon content of interstellar dust towards the Galactic Centre," Mon. Not. R. Astron. Soc. 493, 1109-1119 (2020).

${ }^{54}$ L. P. Keller, S. Bajt, G. A. Baratta, J. Borg, J. P. Bradley, D. E. Brownlee, H. Busemann, J. R. Brucato, M. Burchell, L. Colangeli, L. d'Hendecourt, Z. Djouadi, G. Ferrini, G. Flynn, I. A. Franchi, M. Fries, M. M. Grady, G. A. Graham, F. Grossemy, A. Kearsley, G. Matrajt, K. Nakamura-Messenger, V. Mennella, L. Nittler, M. E. Palumbo, F. J. Stadermann, P. Tsou, A. Rotundi, S. A. Sandford, C. Snead, A. Steele, D. Wooden, and M. Zolensky, "Infrared spectroscopy of comet 81P/wild 2 samples returned by stardust," Science 314, 1728-1731 (2006).

${ }^{55}$ A. Raponi, M. Ciarniello, F. Capaccioni, V. Mennella, G. Filacchione, V. Vinogradoff, O. Poch, P. Beck, E. Quirico, M. C. De Sanctis, L. V. Moroz, D. Kappel, S. Erard, D. Bockelée-Morvan, A. Longobardo, F. Tosi, E. Palomba, J.-P. Combe, B. Rousseau, G. Arnold, R. W. Carlson, A. Pommerol, C. Pilorget, S. Fornasier, G. Bellucci, A. Barucci, F. Mancarella, M. Formisano, G. Rinaldi, I. Istiqomah, and C. Leyrat, "Infrared detection of aliphatic organics on a cometary nucleus," Nat. Astron. 4, 500-505 (2020).

${ }^{56}$ M. Schuhmann, K. Altwegg, H. Balsiger, J.-J. Berthelier, J. De Keyser, B. Fiethe, S. A. Fuselier, S. Gasc, T. I. Gombosi, N. Hänni, M. Rubin, C.-Y. Tzou, and S. F. Wampfler, "Aliphatic and aromatic hydrocarbons in comet 67P/ChuryumovGerasimenko seen by ROSINA," Astron. Astrophys. 630, A31 (2019).

${ }^{57} \mathrm{H}$. Haberland, M. Karrais, and M. Mall, "A new type of cluster and cluster ion source," Z. Phys. D 20, 413-415 (1991).

${ }^{58}$ L. Martínez, M. Díaz, E. Román, M. Ruano, D. Llamosa P., and Y. Huttel, “Generation of nanoparticles with adjustable size and controlled stoichiometry: Recent advances," Langmuir 28, 11241-11249 (2012).

${ }^{59}$ B. L. Frey, R. M. Corn, and S. C. Weibel, "Polarization-modulation approaches to reflection-absorption spectroscopy," in Handbook of Vibrational Spectroscopy (John Wiley \& Sons, Ltd., 2006), pp. 1042-1056.

${ }^{60}$ M. C. Boyer, N. Rivas, A. A. Tran, C. A. Verish, and C. R. Arumainayagam, "The role of low-energy $(\leq 20 \mathrm{eV})$ electrons in astrochemistry," Surf. Sci. 652, 26-32 (2016).

${ }^{61}$ K. E. Shulenberger, J. L. Zhu, K. Tran, S. Abdullahi, C. Belvin, J. Lukens, Z. Peeler, E. Mullikin, H. M. Cumberbatch, J. Huang, K. Regovich, A. Zhou, L. Heller, M. Markovic, L. Gates, C. Buffo, R. Tano-Menka, C. R. Arumainayagam, E. Böhler, P. Swiderek, S. Esmaili, A. D. Bass, M. Huels, and L. Sanche, "Electroninduced radiolysis of astrochemically relevant ammonia ices," ACS Earth Space Chem. 3, 800-810 (2019). 
${ }^{62}$ P. Jenniskens, G. A. Baratta, A. Kouchi, M. S. de Groot, J. M. Greenberg, and G. Strazzulla, "Carbon dust formation on interstellar grains," Astron. Astrophys. 273, 583 (1993).

${ }^{63}$ Y.-J. Chen, K.-J. Chuang, G. M. M. Caro, M. Nuevo, C.-C. Chu, T.-S. Yih, W.H. Ip, and C.-Y. R. Wu, "Vacuum ultraviolet emission spectrum measurement of a microwave-discharge hydrogen-flow lamp in several configurations: Application to photodesorption of CO ice," Astrophys. J. 781, 15 (2014).

${ }^{64} \mathrm{G}$. A. Baratta, G. Leto, and M. E. Palumbo, "A comparison of ion irradiation and UV photolysis of $\mathrm{CH}_{4}$ and $\mathrm{CH}_{3} \mathrm{OH}$," Astron. Astrophys. 384, 343-349 (2002).

${ }^{65}$ D. Fulvio, A. C. Brieva, S. H. Cuylle, H. Linnartz, C. Jäger, and T. Henning, "A straightforward method for vacuum-ultraviolet flux measurements: The case of the hydrogen discharge lamp and implications for solid-phase actinometry," Appl. Phys. Lett. 105, 014105 (2014).

${ }^{66}$ B. D. Teolis, M. Famá, and R. A. Baragiola, “Low density solid ozone," J. Chem. Phys. 127, 074507 (2007).

${ }^{67}$ H. J. Kuhn, S. E. Braslavsky, and R. Schmidt, "Chemical actinometry (IUPAC technical report)," Pure Appl. Chem. 76, 2105-2146 (2004).

${ }^{68}$ R. Martín-Doménech, J. Manzano-Santamaría, G. M. Muñoz Caro, G. A. CruzDíaz, Y.-J. Chen, V. J. Herrero, and I. Tanarro, "UV photoprocessing of $\mathrm{CO}_{2}$ ice: A complete quantification of photochemistry and photon-induced desorption processes," Astron. Astrophys. 584, A14 (2015).
${ }^{69}$ H. Cottin, M. H. Moore, and Y. Benilan, "Photodestruction of relevant interstellar molecules in ice mixtures,” Astrophys. J. 590, 874-881 (2003).

${ }^{70}$ P. A. Gerakines, W. A. Schutte, and P. Ehrenfreund, "Ultraviolet processing of interstellar ice analogs. I. Pure ices,” Astron. Astrophys. 312, 289-305 (1996).

${ }^{71}$ M. Accolla, E. Congiu, F. Dulieu, G. Manicò, H. Chaabouni, E. Matar, H. Mokrane, J. L. Lemaire, and V. Pirronello, "Changes in the morphology of interstellar ice analogues after hydrogen atom exposure," Phys. Chem. Chem. Phys. 13, 8037-8045 (2011)

${ }^{72}$ R. G. Snyder, S. L. Hsu, and S. Krimm, "Vibrational spectra in the C-H stretching region and the structure of the polymethylene chain," Spectrochimi. Acta, Part A 34, 395-406 (1978).

${ }^{73}$ R. G. Snyder and J. H. Schachtschneider, "Vibrational analysis of the $\mathrm{n}-$ paraffins-I: Assignments of infrared bands in the spectra of $\mathrm{C}_{3} \mathrm{H}_{8}$ through n- $\mathrm{C}_{19} \mathrm{H}_{40}$," Spectrochimica Acta 19, 85-116 (1963).

${ }^{74}$ G. Socrates, in Infrared and Raman Characteristic Group Frequencies, edited by J. W. S. Ltd (John Wiley \& Sons, 2001).

${ }^{75}$ J. R. McNesby and H. Okabe, "Vacuum ultraviolet photochemistry," in Advances in Photochemistry (John Wiley \& Sons, Ltd, 2007), pp. 157-240.

${ }^{76}$ NIST Mass Spectrometry Data Center, "Mass spectra," in NIST Chemistry Webbook, NIST Standard Reference Database Number 69 (National Institute of Standards and Technology, 2020). 